\title{
Reviewers - Volume 26
}

The Editors of Atlantic Geology thank the following individuals (and any others whose names may have been inadvertently omitted from the list) for reviewing manuscripts for Volume 26 of the journal.

M.L. Bevier

J. Colwell

G. Dunning

A.S. Dyke

L.E. Fahraeus

D. Fillion

L.R. Fyffe

M.R. Gibling

C.R. Harington

S. Hickock

J.D. Hill

K. Howells

R. Jamieson

D. Kontak

A. Ludman

A. Macdonald

S.R. McCutcheon

H.G. Miller

R.D. Nance

G.M. Narbonne

G. Pe-Piper

D. Prentiss

N. Rast

P. Reynolds

J.E. Riva

A.W.A. Rushton

P.K. Smith

D.B. Stewart

B. VanderPluijm

J. Wallach

R. Wardle

H. Williams

S.H. Williams

R. Wilson

D. Wilton

M. Zentilli 